\title{
The integer part of a nonlinear form with integer variables
}

Kai Lai*

${ }^{\text {*Correspondence: }}$

k.ching.lai@foxmail.com

College of Computer and

Information Engineering, Henan

University of Economics and Law

Zhengzhou, 450011, P.R. China

\begin{abstract}
Using the Davenport-Heilbronn method, we show that if $\lambda_{1}, \lambda_{2}, \ldots, \lambda_{9}$ are positive real numbers, at least one of the ratios $\lambda_{j} / \lambda_{j}(1 \leq i<j \leq 9)$ is irrational, then the integer parts of $\lambda_{1} x_{1}^{3}+\lambda_{2} x_{2}^{3}+\lambda_{3} x_{3}^{4}+\lambda_{4} x_{4}^{4}+\lambda_{5} x_{5}^{5}+\cdots+\lambda_{9} x_{9}^{5}$ are prime infinitely often for natural numbers $x_{1}, x_{2}, \ldots, x_{9}$.
\end{abstract}

Keywords: Davenport-Heilbronn method; integer variables; diophantine approximation

\section{Introduction}

In 2010, Brüdern et al. [1] proved that if $\lambda_{1}, \ldots, \lambda_{s}$ are positive real numbers, $\lambda_{1} / \lambda_{2}$ is irrational, all Dirichlet $L$-functions satisfy the Riemann hypothesis $s \geq \frac{8}{3} k+2$, then the integer parts of

$$
\lambda_{1} x_{1}^{k}+\lambda_{2} x_{2}^{k}+\cdots+\lambda_{s} x_{s}^{k}
$$

are prime infinitely often for natural numbers $x_{j}$.

Motivated by [1], using the Davenport-Heilbronn method, we consider the integer part of a nonlinear form with integer variables and mixed powers 3,4 and 5 , and establish one result as follows.

Theorem 1.1 Let $\lambda_{1}, \lambda_{2}, \ldots, \lambda_{9}$ be positive real numbers, at least one of the ratios $\lambda_{i} / \lambda_{j}(1 \leq$ $i<j \leq 9)$ is irrational. Then the integer parts of

$$
\lambda_{1} x_{1}^{3}+\lambda_{2} x_{2}^{3}+\lambda_{3} x_{3}^{4}+\lambda_{4} x_{4}^{4}+\lambda_{5} x_{5}^{5}+\cdots+\lambda_{9} x_{9}^{5}
$$

are prime infinitely often for natural numbers $x_{1}, x_{2}, \ldots, x_{9}$.

It is noted that Theorem 1.1 holds without the Riemann hypothesis.

\section{Notation}

Throughout, we use $p$ to denote a prime number and $x_{j}$ to denote a natural number. We denote by $\delta$ a sufficiently small positive number and by $\varepsilon$ an arbitrarily small positive number. Constants, both explicit and implicit, in Landau or Vinogradov symbols may depend

(c) 2015 Lai. This article is distributed under the terms of the Creative Commons Attribution 4.0 International License (http://creativecommons.org/licenses/by/4.0/), which permits unrestricted use, distribution, and reproduction in any medium, provided you give appropriate credit to the original author(s) and the source, provide a link to the Creative Commons license, and indicate if changes were made. 
on $\lambda_{1}, \lambda_{2}, \ldots, \lambda_{9}$. We write $e(x)=\exp (2 \pi i x)$. We use $[x]$ to denote the integer part of real variable $x$. We take $X$ to be the basic parameter, a large real integer. Since at least one of the ratios $\lambda_{i} / \lambda_{j}(1 \leq i<j \leq 9)$ is irrational, without loss of generality we may assume that $\lambda_{1} / \lambda_{2}$ is irrational. For the other cases, the only difference is in the following intermediate region, and we may deal with the same method in Section 4.

Since $\lambda_{1} / \lambda_{2}$ is irrational, then there are infinitely many pairs of integers $q$, $a$ with $\mid \lambda_{1} / \lambda_{2}-$ $a / q \mid \leq q^{-2},(a, q)=1, q>0$ and $a \neq 0$. We choose $q$ to be large in terms of $\lambda_{1}, \lambda_{2}, \ldots, \lambda_{9}$ and make the following definitions.

$$
\begin{array}{lcc}
N \asymp X, \quad L=\log N, & {\left[N^{1-8 \delta}\right]=q,} & \tau=N^{-1+\delta}, \\
Q=\left(\left|\lambda_{1}\right|^{-1}+\left|\lambda_{2}\right|^{-1}\right) N^{1-\delta}, & P=N^{6 \delta}, & T=N^{\frac{1}{3}} .
\end{array}
$$

Let $v$ be a positive real number, we define

$$
\begin{aligned}
& K_{v}(\alpha)=v\left(\frac{\sin \pi v \alpha}{\pi v \alpha}\right)^{2}, \quad \alpha \neq 0, \quad K_{v}(0)=v, \\
& F_{i}(\alpha)=\sum_{1 \leq x \leq X^{\frac{1}{3}}} e\left(\alpha x^{3}\right), \quad i=1,2, \\
& F_{j}(\alpha)=\sum_{1 \leq x \leq X^{\frac{1}{4}}} e\left(\alpha x^{4}\right), \quad j=3,4, \\
& F_{k}(\alpha)=\sum_{1 \leq x \leq X^{\frac{1}{5}}} e\left(\alpha x^{5}\right), \quad k=5, \ldots, 9, \\
& G(\alpha)=\sum_{p \leq N}(\log p) e(\alpha p), \\
& f_{i}(\alpha)=\int_{1}^{X^{\frac{1}{3}}} e\left(\alpha x^{3}\right) d x, \quad i=1,2, \\
& f_{j}(\alpha)=\int_{1}^{X^{\frac{1}{4}}} e\left(\alpha x^{4}\right) d x, \quad j=3,4, \\
& f_{k}(\alpha)=\int_{1}^{X^{\frac{1}{5}}} e\left(\alpha x^{5}\right) d x, \quad k=5, \ldots, 9, \\
& g(\alpha)=\int_{1}^{N} e(\alpha x) d x .
\end{aligned}
$$

It follows from (2.1) that

$$
\begin{aligned}
& K_{v}(\alpha) \ll \min \left(v, v^{-1}|\alpha|^{-2}\right), \\
& \int_{-\infty}^{+\infty} e(\alpha y) K_{v}(\alpha) d \alpha=\max \left(0,1-v^{-1}|y|\right) .
\end{aligned}
$$

From (2.3) it is clear that 


$$
\begin{aligned}
J & =: \int_{-\infty}^{+\infty} \prod_{i=1}^{9} F_{i}\left(\lambda_{i} \alpha\right) G(-\alpha) e\left(-\frac{1}{2} \alpha\right) K_{\frac{1}{2}}(\alpha) d \alpha \\
& \leq \log N \sum_{\substack{\left|\lambda_{1} x_{1}^{3}+\lambda_{2} x_{2}^{3}+\lambda_{3} x_{3}^{4}+\lambda_{4} x_{4}^{4}+\lambda_{5} x_{5}^{5}+\cdots+\lambda_{9} x_{9}^{5}-p-\frac{1}{2}\right|<\frac{1}{2} \\
1 \leq x_{1}, x_{2} \leq X^{1 / 3}, 1 \leq x_{3}, x_{4} \leq X^{1 / 4}, 1 \leq x_{5}, \ldots, x_{9} \leq X^{1 / 5}, p \leq N}} 1 \\
& =:(\log N) \mathcal{N}(X),
\end{aligned}
$$

thus

$$
\mathcal{N}(X) \geq(\log N)^{-1} J
$$

To estimate $J$, we split the range of infinite integration into three sections, traditional named the neighborhood of the origin $\mathfrak{C}=\{\alpha \in \mathbb{R}:|\alpha| \leq \tau\}$, the intermediate region $\mathfrak{D}=$ $\{\alpha \in \mathbb{R}: \tau<|\alpha| \leq P\}$ and the trivial region $\mathfrak{c}=\{\alpha \in \mathbb{R}:|\alpha|>P\}$.

\section{The neighborhood of the origin}

Lemma 3.1 If $\alpha=a / q+\beta$, where $(a, q)=1$, then

$$
\sum_{1 \leq x \leq N^{1 / t}} e\left(\alpha x^{t}\right)=q^{-1} \sum_{m=1}^{q} e\left(a m^{t} / q\right) \int_{1}^{N^{1 / t}} e\left(\beta y^{t}\right) d y+O\left(q^{1 / 2+\varepsilon}(1+N|\beta|)\right) .
$$

Proof This is Theorem 4.1 of [2].

If $|\alpha| \in \mathfrak{C}$, by Lemma 3.1, taking $a=0, q=1$, then

$$
F_{i}(\alpha)=f_{i}(\alpha)+O\left(X^{\delta}\right), \quad i=1,2, \ldots, 9 .
$$

Lemma 3.2 Let $\rho=\beta+i \gamma$ be a typical zero of the Riemann zeta function, $C$ be a positive constant,

$$
I(\alpha)=\sum_{|\gamma| \leq T, \beta \geq \frac{2}{3}} \sum_{n \leq N} n^{\rho-1} e(n \alpha), \quad J(\alpha)=O\left((1+|\alpha| N) N^{\frac{2}{3}} L^{C}\right),
$$

then

$$
\begin{aligned}
& G(\alpha)=g(\alpha)-I(\alpha)+J(\alpha), \\
& \int_{-\frac{1}{2}}^{\frac{1}{2}}|I(\alpha)|^{2} d \alpha \ll N \exp \left(-L^{\frac{1}{5}}\right), \\
& \int_{-\tau}^{\tau}|J(\alpha)|^{2} d \alpha \ll N \exp \left(-L^{\frac{1}{5}}\right) .
\end{aligned}
$$

Proof Equations (3.2), (3.3), (3.4) can be seen from Lemma 5, (29) and (33) given by Vaughan [3]. 
Lemma 3.3 We have

$$
\begin{aligned}
& \int_{-\frac{1}{2}}^{\frac{1}{2}}\left|f_{i}(\alpha)\right|^{2} d \alpha \ll X^{-\frac{1}{3}}, \quad i=1,2, \\
& \int_{-\frac{1}{2}}^{\frac{1}{2}}\left|f_{j}(\alpha)\right|^{2} d \alpha \ll X^{-\frac{1}{2}}, \quad j=3,4, \\
& \int_{-\frac{1}{2}}^{\frac{1}{2}}\left|f_{k}(\alpha)\right|^{2} d \alpha \ll X^{-\frac{3}{5}}, \quad k=5, \ldots, 9 .
\end{aligned}
$$

Proof These results are from Lemma 5 of [3].

Lemma 3.4 We have

$$
\int_{\mathfrak{C}}\left|\prod_{i=1}^{9} F_{i}\left(\lambda_{i} \alpha\right) G(-\alpha)-\prod_{i=1}^{9} f_{i}\left(\lambda_{i} \alpha\right) g(-\alpha)\right| K_{\frac{1}{2}}(\alpha) d \alpha \ll X^{\frac{13}{6}} L^{-1}
$$

Proof It is obvious that

$$
\begin{aligned}
& F_{i}\left(\lambda_{i} \alpha\right) \ll X^{\frac{1}{3}}, \quad f_{i}\left(\lambda_{i} \alpha\right) \ll X^{\frac{1}{3}}, \quad i=1,2, \\
& F_{j}\left(\lambda_{j} \alpha\right) \ll X^{\frac{1}{4},} \quad f_{j}\left(\lambda_{j} \alpha\right) \ll X^{\frac{1}{4}}, \quad j=3,4, \\
& F_{k}\left(\lambda_{k} \alpha\right) \ll X^{\frac{1}{5}}, \quad f_{k}\left(\lambda_{k} \alpha\right) \ll X^{\frac{1}{5}}, \quad k=5, \ldots, 9, \\
& G(-\alpha) \ll N, \quad g(-\alpha) \ll N, \\
& \prod_{i=1}^{9} F_{i}\left(\lambda_{i} \alpha\right) G(-\alpha)-\prod_{i=1}^{9} f_{i}\left(\lambda_{i} \alpha\right) g(-\alpha) \\
& =\left(F_{1}\left(\lambda_{1} \alpha\right)-f_{1}\left(\lambda_{1} \alpha\right)\right) \prod_{i=2}^{9} F_{i}\left(\lambda_{i} \alpha\right) G(-\alpha) \\
& \quad+\left(F_{2}\left(\lambda_{2} \alpha\right)-f_{2}\left(\lambda_{2} \alpha\right)\right) \prod_{\substack{i=1 \\
i \neq 2}}^{9} F_{i}\left(\lambda_{i} \alpha\right) G(-\alpha)+\cdots \\
& \quad+\left(F_{9}\left(\lambda_{9} \alpha\right)-f_{9}\left(\lambda_{9} \alpha\right)\right) \prod_{i=1}^{8} f_{i}\left(\lambda_{i} \alpha\right) G(-\alpha)+\prod_{i=1}^{9} f_{i}\left(\lambda_{i} \alpha\right)(G(-\alpha)-g(-\alpha)) .
\end{aligned}
$$

Then by (3.1), Lemmas 3.2 and 3.3, we have

$$
\begin{aligned}
& \int_{\mathfrak{C}}\left|\left(F_{1}\left(\lambda_{1} \alpha\right)-f_{1}\left(\lambda_{1} \alpha\right)\right) \prod_{i=2}^{9} F_{i}\left(\lambda_{i} \alpha\right) G(-\alpha)\right| K_{\frac{1}{2}}(\alpha) d \alpha \ll N^{-1+\delta} X^{\delta} X^{\frac{11}{6}} N \ll X^{\frac{11}{6}+2 \delta}, \\
& \int_{\mathfrak{C}}\left|\prod_{i=1}^{9} f_{i}\left(\lambda_{i} \alpha\right)(G(-\alpha)-g(-\alpha))\right| K_{\frac{1}{2}}(\alpha) d \alpha \\
& \ll X^{\frac{11}{6}}\left(\int_{\mathfrak{C}}\left|f_{1}\left(\lambda_{1} \alpha\right)\right|^{2} K_{\frac{1}{2}}(\alpha) d \alpha\right)^{\frac{1}{2}}\left(\int_{\mathfrak{C}}|J(-\alpha)-I(-\alpha)|^{2} K_{\frac{1}{2}}(\alpha) d \alpha\right)^{\frac{1}{2}}
\end{aligned}
$$




$$
\begin{aligned}
& \ll X^{\frac{11}{6}}\left(\int_{-\frac{1}{2}}^{\frac{1}{2}}\left|f_{1}\left(\lambda_{1} \alpha\right)\right|^{2} d \alpha\right)^{\frac{1}{2}}\left(\int_{\mathfrak{C}}|J(\alpha)|^{2} d \alpha+\int_{-\frac{1}{2}}^{\frac{1}{2}}|I(\alpha)|^{2} d \alpha\right)^{\frac{1}{2}} \\
& \ll X^{\frac{11}{6}} X^{-\frac{1}{6}}\left(N \exp \left(-L^{\frac{1}{5}}\right)\right)^{\frac{1}{2}} \\
& \ll X^{\frac{13}{6}} L^{-1} .
\end{aligned}
$$

The other cases are similar, and the proof of Lemma 3.4 is completed.

Lemma 3.5 We have

$$
\int_{|\alpha|>N^{-1+\delta}}\left|\prod_{i=1}^{9} f_{i}\left(\lambda_{i} \alpha\right) g(-\alpha)\right| K_{\frac{1}{2}}(\alpha) d \alpha \ll X^{\frac{13}{6}-\frac{13}{6} \delta} .
$$

Proof It follows from Vaughan [2] that for $\alpha \neq 0$,

$$
\begin{aligned}
& f_{i}\left(\lambda_{i} \alpha\right) \ll|\alpha|^{-\frac{1}{3}}, \quad i=1,2, \quad f_{j}\left(\lambda_{j} \alpha\right) \ll|\alpha|^{-\frac{1}{4}}, \quad j=3,4, \\
& f_{k}\left(\lambda_{k} \alpha\right) \ll|\alpha|^{-\frac{1}{5}}, \quad k=5, \ldots, 9, \quad g(-\alpha) \ll|\alpha|^{-1} .
\end{aligned}
$$

Thus

$$
\int_{|\alpha|>N^{-1+\delta}}\left|\prod_{i=1}^{9} f_{i}\left(\lambda_{i} \alpha\right) g(-\alpha)\right| K_{\frac{1}{2}}(\alpha) d \alpha \ll \int_{|\alpha|>N^{-1+\delta}}|\alpha|^{-\frac{19}{6}} d \alpha \ll X^{\frac{13}{6}-\frac{13}{6} \delta} .
$$

Lemma 3.6 We have

$$
\int_{-\infty}^{+\infty} \prod_{i=1}^{9} f_{i}\left(\lambda_{i} \alpha\right) g(-\alpha) e\left(-\frac{1}{2} \alpha\right) K_{\frac{1}{2}}(\alpha) d \alpha \gg X^{\frac{13}{6}} .
$$

Proof From (2.3) one has

$$
\begin{aligned}
\int_{-\infty}^{+\infty} & \prod_{i=1}^{9} f_{i}\left(\lambda_{i} \alpha\right) g(-\alpha) e\left(-\frac{1}{2} \alpha\right) K_{\frac{1}{2}}(\alpha) d \alpha \\
= & \int_{1}^{X^{\frac{1}{3}}} \int_{1}^{X^{\frac{1}{3}}} \int_{1}^{X^{\frac{1}{4}}} \int_{1}^{X^{\frac{1}{4}}} \int_{1}^{X^{\frac{1}{5}}} \cdots \int_{1}^{X^{\frac{1}{5}}} \int_{1}^{N} \int_{-\infty}^{+\infty} e\left(\alpha \left(\lambda_{1} x_{1}^{3}+\lambda_{2} x_{2}^{3}+\lambda_{3} x_{3}^{4}+\lambda_{4} x_{4}^{4}\right.\right. \\
& \left.\left.+\lambda_{5} x_{5}^{5}+\cdots+\lambda_{9} x_{9}^{5}-x-\frac{1}{2}\right)\right) K_{\frac{1}{2}}(\alpha) d \alpha d x d x_{9} \cdots d x_{5} d x_{4} d x_{3} d x_{2} d x_{1} \\
= & \frac{1}{450,000} \int_{1}^{X} \cdots \int_{1}^{X} \int_{1}^{N} \int_{-\infty}^{+\infty} x_{1}^{-\frac{2}{3}} x_{2}^{-\frac{2}{3}} x_{3}^{-\frac{3}{4}} x_{4}^{-\frac{3}{4}} x_{5}^{-\frac{4}{5}} \cdots x_{9}^{-\frac{4}{5}} e\left(\alpha\left(\sum_{i=1}^{9} \lambda_{i} x_{i}-x-\frac{1}{2}\right)\right) \\
& \cdot K_{\frac{1}{2}}(\alpha) d \alpha d x d x_{9} \cdots d x_{1} \\
= & \frac{1}{450,000} \int_{1}^{X} \cdots \int_{1}^{X} \int_{1}^{N} x_{1}^{-\frac{2}{3}} x_{2}^{-\frac{2}{3}} x_{3}^{-\frac{3}{4}} x_{4}^{-\frac{3}{4}} x_{5}^{-\frac{4}{5}} \cdots x_{9}^{-\frac{4}{5}} \\
& \cdot \max \left(0, \frac{1}{2}-\left|\sum_{i=1}^{9} \lambda_{i} x_{i}-x-\frac{1}{2}\right|\right) d x d x_{9} \cdots d x_{1} .
\end{aligned}
$$


Let $\left|\sum_{i=1}^{9} \lambda_{i} x_{i}-x-\frac{1}{2}\right| \leq \frac{1}{4}$, then $\sum_{i=1}^{9} \lambda_{i} x_{i}-\frac{3}{4} \leq x \leq \sum_{i=1}^{9} \lambda_{i} x_{i}-\frac{1}{4}$. Based on

$$
\sum_{i=1}^{9} \lambda_{i} x_{i}-\frac{3}{4}>1, \quad \sum_{i=1}^{9} \lambda_{i} x_{i}-\frac{1}{4}<N
$$

one may take

$$
\lambda_{j} X\left(8 \sum_{i=1}^{9} \lambda_{i}\right)^{-1} \leq x_{j} \leq \lambda_{j} X\left(4 \sum_{i=1}^{9} \lambda_{i}\right)^{-1}, \quad j=1, \ldots, 9,
$$

hence

$$
\int_{-\infty}^{+\infty} \prod_{i=1}^{9} f_{i}\left(\lambda_{i} \alpha\right) g(-\alpha) e\left(-\frac{1}{2} \alpha\right) K_{\frac{1}{2}}(\alpha) d \alpha \geq \frac{1}{3,600,000} \prod_{j=1}^{9} \lambda_{j}\left(8 \sum_{i=1}^{9} \lambda_{i}\right)^{-9} X^{\frac{13}{6}}
$$

This completes the proof of Lemma 3.6.

\section{The intermediate region}

\section{Lemma 4.1 We have}

$$
\begin{aligned}
& \int_{-\infty}^{+\infty}\left|F_{i}\left(\lambda_{i} \alpha\right)\right|^{8} K_{\frac{1}{2}}(\alpha) d \alpha \ll X^{\frac{5}{3}+\frac{1}{3} \varepsilon}, \quad i=1,2, \\
& \int_{-\infty}^{+\infty}\left|F_{j}\left(\lambda_{j} \alpha\right)\right|^{16} K_{\frac{1}{2}}(\alpha) d \alpha \ll X^{3+\frac{1}{4} \varepsilon}, \quad j=3,4, \\
& \int_{-\infty}^{+\infty}\left|F_{k}\left(\lambda_{k} \alpha\right)\right|^{32} K_{\frac{1}{2}}(\alpha) d \alpha \ll X^{\frac{27}{5}+\frac{1}{5} \varepsilon}, \quad k=5, \ldots, 9, \\
& \int_{-\infty}^{+\infty}|G(-\alpha)|^{2} K_{\frac{1}{2}}(\alpha) d \alpha \ll N L .
\end{aligned}
$$

Proof By (2.2) and Hua's inequality, for $i=1,2$, we have

$$
\begin{aligned}
& \int_{-\infty}^{+\infty}\left|F_{i}\left(\lambda_{i} \alpha\right)\right|^{8} K_{\frac{1}{2}}(\alpha) d \alpha \\
& \ll \sum_{m=-\infty}^{+\infty} \int_{m}^{m+1}\left|F_{i}\left(\lambda_{i} \alpha\right)\right|^{8} K_{\frac{1}{2}}(\alpha) d \alpha \\
& \ll \sum_{m=0}^{1} \int_{m}^{m+1}\left|F_{i}\left(\lambda_{i} \alpha\right)\right|^{8} d \alpha+\sum_{m=2}^{+\infty} m^{-2} \int_{m}^{m+1}\left|F_{i}\left(\lambda_{i} \alpha\right)\right|^{8} d \alpha \\
& \ll X^{\frac{5}{3}+\frac{1}{3} \varepsilon}+X^{\frac{5}{3}+\frac{1}{3} \varepsilon} \sum_{m=2}^{+\infty} m^{-2} \\
& \ll X^{\frac{5}{3}+\frac{1}{3} \varepsilon} .
\end{aligned}
$$

The proofs of (4.2)-(4.4) are similar to (4.1). 
Lemma 4.2 Suppose that $(a, q)=1,|\alpha-a / q| \leq q^{-2}, \phi(x)=\alpha x^{k}+\alpha_{1} x^{k-1}+\cdots+\alpha_{k-1} x+\alpha_{k}$, then

$$
\sum_{x=1}^{M} e(\phi(x)) \ll M^{1+\varepsilon}\left(q^{-1}+M^{-1}+q M^{-k}\right)^{2^{1-k}}
$$

Proof This is Lemma 2.4 (Weyl's inequality) of Vaughan [2].

Lemma 4.3 For every real number $\alpha \in \mathfrak{D}$, let $W(\alpha)=\min \left(\left|F_{1}\left(\lambda_{1} \alpha\right)\right|,\left|F_{2}\left(\lambda_{2} \alpha\right)\right|\right)$, then

$$
W(\alpha) \ll X^{\frac{1}{3}-\frac{1}{4} \delta+\frac{1}{3} \varepsilon}
$$

Proof For $\alpha \in \mathfrak{D}$ and $i=1,2$, we choose $a_{i}, q_{i}$ such that

$$
\left|\lambda_{i} \alpha-a_{i} / q_{i}\right| \leq q_{i}^{-1} Q^{-1}
$$

with $\left(a_{i}, q_{i}\right)=1$ and $1 \leq q_{i} \leq Q$.

Firstly, we note that $a_{1} a_{2} \neq 0$. Secondly, if $q_{1}, q_{2} \leq P$, then

$$
\left|a_{2} q_{1} \frac{\lambda_{1}}{\lambda_{2}}-a_{1} q_{2}\right| \leq\left|\frac{a_{2} / q_{2}}{\lambda_{2} \alpha} q_{1} q_{2}\left(\lambda_{1} \alpha-\frac{a_{1}}{q_{1}}\right)\right|+\left|\frac{a_{1} / q_{1}}{\lambda_{2} \alpha} q_{1} q_{2}\left(\lambda_{2} \alpha-\frac{a_{2}}{q_{2}}\right)\right| \ll P Q^{-1}<\frac{1}{2 q} .
$$

We recall that $q$ was chosen as the denominator of a convergent to the continued fraction for $\lambda_{1} / \lambda_{2}$. Thus, by Legendre's law of best approximation, we have $\left|q^{\prime} \frac{\lambda_{1}}{\lambda_{2}}-a^{\prime}\right|>\frac{1}{2 q}$ for all integers $a^{\prime}, q^{\prime}$ with $1 \leq q^{\prime}<q$, thus $\left|a_{2} q_{1}\right| \geq q=\left[N^{1-8 \delta}\right]$. However, from (4.5) we have $\left|a_{2} q_{1}\right| \ll q_{1} q_{2} P \ll N^{18 \delta}$, this is a contradiction. We have thus established that for at least one $i, P<q_{i} \ll Q$. Hence Lemma 4.2 gives the desired inequality for $W(\alpha)$.

Lemma 4.4 We have

$$
\int_{\mathfrak{D}} \prod_{i=1}^{9} F_{i}\left(\lambda_{i} \alpha\right) G(-\alpha) e\left(-\frac{1}{2} \alpha\right) K_{\frac{1}{2}}(\alpha) d \alpha \ll X^{\frac{13}{6}-\frac{1}{16} \delta+\varepsilon}
$$

Proof By Lemmas 4.1, 4.3 and Hölder's inequality, we have

$$
\begin{aligned}
\int_{\mathfrak{D}} & \prod_{i=1}^{9}\left|F_{i}\left(\lambda_{i} \alpha\right) G(-\alpha)\right| K_{\frac{1}{2}}(\alpha) d \alpha \\
\ll & \max _{\alpha \in \mathfrak{D}}|W(\alpha)|^{\frac{1}{4}}\left(\left(\int_{-\infty}^{+\infty}\left|F_{1}\left(\lambda_{1} \alpha\right)\right|^{8}\right)^{\frac{1}{8}}\left(\int_{-\infty}^{+\infty}\left|F_{2}\left(\lambda_{2} \alpha\right)\right|^{8}\right)^{\frac{3}{32}}\right. \\
& \left.+\left(\int_{-\infty}^{+\infty}\left|F_{1}\left(\lambda_{1} \alpha\right)\right|^{8}\right)^{\frac{3}{32}}\left(\int_{-\infty}^{+\infty}\left|F_{2}\left(\lambda_{2} \alpha\right)\right|^{8}\right)^{\frac{1}{8}}\right) \\
& \cdot\left(\prod_{j=3}^{4} \int_{-\infty}^{+\infty}\left|F_{j}\left(\lambda_{j} \alpha\right)\right|^{16} K_{\frac{1}{2}}(\alpha) d \alpha\right)^{\frac{1}{16}}\left(\prod_{k=5}^{9} \int_{-\infty}^{+\infty}\left|F_{k}\left(\lambda_{k} \alpha\right)\right|^{32} K_{\frac{1}{2}}(\alpha) d \alpha\right)^{\frac{1}{32}} \\
& \cdot\left(\int_{-\infty}^{+\infty}|G(-\alpha)|^{2} K_{\frac{1}{2}}(\alpha) d \alpha\right)^{\frac{1}{2}}
\end{aligned}
$$




$$
\begin{aligned}
& \ll\left(X^{\frac{1}{3}-\frac{1}{4} \delta+\frac{1}{3} \varepsilon}\right)^{\frac{1}{4}}\left(X^{\frac{5}{3}+\frac{1}{3} \varepsilon}\right)^{\frac{7}{32}}\left(X^{3+\frac{1}{4} \varepsilon}\right)^{\frac{1}{8}}\left(X^{\frac{27}{5}+\frac{1}{5} \varepsilon}\right)^{\frac{5}{32}}(N L)^{\frac{1}{2}} \\
& \ll X^{\frac{13}{6}-\frac{1}{16} \delta+\varepsilon} .
\end{aligned}
$$

\section{The trivial region}

Lemma 5.1 (Lemma 2 of [4]) Let $V(\alpha)=\sum e\left(\alpha f\left(x_{1}, \ldots, x_{m}\right)\right)$, where $f$ is any real function and the summation is over any finite set of values of $x_{1}, \ldots, x_{m}$. Then, for any $A>4$, we have

$$
\int_{|\alpha|>A}|V(\alpha)|^{2} K_{v}(\alpha) d \alpha \leq \frac{16}{A} \int_{-\infty}^{\infty}|V(\alpha)|^{2} K_{v}(\alpha) d \alpha .
$$

Lemma 5.2 We have

$$
\int_{\mathfrak{c}} \prod_{i=1}^{9} F_{i}\left(\lambda_{i} \alpha\right) G(-\alpha) e\left(-\frac{1}{2} \alpha\right) K_{\frac{1}{2}}(\alpha) d \alpha \ll X^{\frac{13}{6}-6 \delta+\varepsilon} .
$$

Proof By Lemmas 5.1, 4.1 and Schwarz's inequality, we have

$$
\begin{aligned}
\int_{\mathfrak{c}} & \prod_{i=1}^{9} F_{i}\left(\lambda_{i} \alpha\right) G(-\alpha) e\left(-\frac{1}{2} \alpha\right) K_{\frac{1}{2}}(\alpha) d \alpha \\
\ll & \int_{\mathfrak{c}}\left|\prod_{i=1}^{9} F_{i}\left(\lambda_{i} \alpha\right) G(-\alpha)\right| K_{\frac{1}{2}}(\alpha) d \alpha \\
\ll & \frac{1}{P} \int_{-\infty}^{+\infty}\left|\prod_{i=1}^{9} F_{i}\left(\lambda_{i} \alpha\right) G(-\alpha)\right| K_{\frac{1}{2}}(\alpha) d \alpha \\
\ll & N^{-6 \delta}\left|F_{1}\left(\lambda_{1} \alpha\right)\right|^{\frac{1}{4}}\left(\left(\int_{-\infty}^{+\infty}\left|F_{1}\left(\lambda_{1} \alpha\right)\right|^{8}\right)^{\frac{3}{32}}\left(\int_{-\infty}^{+\infty}\left|F_{2}\left(\lambda_{2} \alpha\right)\right|^{8}\right)^{\frac{1}{8}}\right) \\
& \cdot\left(\prod_{j=3}^{4} \int_{-\infty}^{+\infty}\left|F_{j}\left(\lambda_{j} \alpha\right)\right|^{16} K_{\frac{1}{2}}(\alpha) d \alpha\right)^{\frac{1}{16}}\left(\prod_{k=5}^{9} \int_{-\infty}^{+\infty} \mid F_{k}\left(\left.\lambda_{k} \alpha\right|^{32} K_{\frac{1}{2}}(\alpha) d \alpha\right)^{\frac{1}{32}}\right. \\
& \cdot\left(\int_{-\infty}^{+\infty}|G(-\alpha)|^{2} K_{\frac{1}{2}}(\alpha) d \alpha\right)^{\frac{1}{2}} \\
\ll & N^{-6 \delta}\left(X^{\frac{1}{3}}\right)^{\frac{1}{4}}\left(X^{\frac{5}{3}+\frac{1}{3} \varepsilon}\right)^{\frac{7}{32}}\left(X^{3+\frac{1}{4} \varepsilon}\right)^{\frac{1}{8}}\left(X^{\frac{27}{5}+\frac{1}{5} \varepsilon}\right)^{\frac{5}{32}}(N L)^{\frac{1}{2}} \\
\ll & X^{\frac{13}{6}-6 \delta+\varepsilon} .
\end{aligned}
$$

\section{The proof of Theorem 1.1}

From Lemmas 3.4, 3.5 and 3.6 we conclude that $J(\mathfrak{C}) \gg X^{\frac{13}{6}}$. From Lemma 4.4 it follows that $J(\mathfrak{D})=o\left(X^{\frac{13}{6}}\right)$. From Lemma 5.2 we have $J(\mathfrak{c})=o\left(X^{\frac{13}{6}}\right)$. Thus

$$
J \gg X^{\frac{13}{6}}, \quad \mathcal{N}(X) \gg X^{\frac{13}{6}} L^{-1}
$$

namely, under conditions of Theorem 1.1,

$$
\left|\lambda_{1} x_{1}^{3}+\lambda_{2} x_{2}^{3}+\lambda_{3} x_{3}^{4}+\lambda_{4} x_{4}^{4}+\lambda_{5} x_{5}^{5}+\cdots+\lambda_{9} x_{9}^{5}-p-\frac{1}{2}\right|<\frac{1}{2}
$$


has infinitely many solutions in positive integers $x_{1}, x_{2}, \ldots, x_{9}$ and prime $p$. It is evident from (6.1) that

$$
p<\lambda_{1} x_{1}^{3}+\lambda_{2} x_{2}^{3}+\lambda_{3} x_{3}^{4}+\lambda_{4} x_{4}^{4}+\lambda_{5} x_{5}^{5}+\cdots+\lambda_{9} x_{9}^{5}<p+1,
$$

and hence

$$
\left[\lambda_{1} x_{1}^{3}+\lambda_{2} x_{2}^{3}+\lambda_{3} x_{3}^{4}+\lambda_{4} x_{4}^{4}+\lambda_{5} x_{5}^{5}+\cdots+\lambda_{9} x_{9}^{5}\right]=p
$$

The proof of Theorem 1.1 is complete.

\section{Competing interests}

The author declares that he has no competing interests.

Received: 30 August 2015 Accepted: 28 October 2015 Published online: 11 November 2015

References

1. Brüdern, J, Kawada, K, Wooley, TD: Additive representation in thin sequences, VIII: Diophantine inequalities in review. In: Number Theory: Dreaming in Dreams. Series on Number Theory and Its Applications, vol. 6, pp. 20-79 (2010)

2. Vaughan, R: The Hardy-Littlewood Method, 2nd edn. Cambridge Tracts in Mathematics, vol. 125. Cambridge University Press, Cambridge (1997)

3. Vaughan, R: Diophantine approximation by prime numbers I. Proc. Lond. Math. Soc. 28, 373-384 (1974)

4. Davenport, H, Roth, KF: The solubility of certain Diophantine inequalities. Mathematika 2, 81-96 (1955)

\section{Submit your manuscript to a SpringerOpen ${ }^{\circ}$ journal and benefit from:}

- Convenient online submission

Rigorous peer review

- Immediate publication on acceptance

Open access: articles freely available online

- High visibility within the field

- Retaining the copyright to your article 